\title{
January 2014 Tucson Critical Care Journal Club: Esmolol in Septic Shock
}

\section{Morelli A, Ertmer C, Westphal M, et al. Effect of heart rate control with esmolol on hemodynamic and clinical outcomes in patients with septic shock: a randomized clinical trial. JAMA. 2013;310(16):1683-91. [CrossRef] [PubMed]}

During septic shock, catecholamines serve to elevate heart rate and maintain cardiac output as an adaptive response to low peripheral vascular resistance. However, elevated serum catecholamines and tachycardia are associated with poor outcomes in the intensive care unit (ICU) (1). This study hypothesized that $\beta$-blocker therapy (intravenous esmolol) titrated to control heart rate would enhance myocardial function and improve outcomes in patients with septic shock.

This single center, randomized, open label, parallel group study randomized 154 participants to either receive intravenous esmolol titrated to achieve a heart rate of 8094 beats per minute (bpm) or standard of care. Randomization occurred after 24 hours of hemodynamic optimization, including appropriate volume resuscitation. Inclusion criteria included the presence of septic shock requiring norepinephrine, heart rate $>95$ $\mathrm{bpm}$ and age $>18$. Exclusion criteria included a heart rate $<95$ at randomization, significant cardiac or valvular dysfunction and pregnancy. The primary outcome was the difference in heart rate as measured by the mean area under the curves (AUCs) during the first 96 hours of treatment. Secondary outcomes included norepinephrine requirements, cardiopulmonary and oxygen indices, safety endpoints, and 28-day mortality.

Baseline patient characteristics were similar between the two treatment groups. Of note, there was an unusually high rate of multidrug-resistant Klebsiella and Acinetobacter baumannii among both groups. SAPS II scores were calculated based on variables at the time of randomization and were similar between the groups.

Compared to the control group, the intervention group achieved a lower median AUC for $\mathrm{HR}(\mathrm{P}<.001)$ with all intervention group participants meeting the heart rate goal of 80 $94 \mathrm{bpm}$. The intervention group had a 28 -day mortality of $49.4 \%$ vs. $80.5 \%$ in the control group $(P<.001$, hazard ratio, 0.392$)$. Intervention group median AUC for norepinephrine requirements were significant less $(P<.003)$ without a corresponding decrease in mean arterial pressure. Median AUC was significantly higher in the intervention group compared to the control group for stroke volume index $(P<.02)$, systemic vascular resistance index $(\mathrm{P}<.001)$, left ventricular stroke work index $(\mathrm{P}=$ .03), GFR $(P<.001)$, and $\mathrm{PaO}_{2} / \mathrm{FiO}_{2}(P=.03)$. Lastly, the intervention group median $A \cup C$ for fluid requirements were significantly less $(P<.001)$.

Although this was a well-designed study, there are several limitations to consider. First, the study was nonblinded, raising concern for unconscious bias in delivery of care. Second, there was an unusually high rate of multidrug-resistant Klebsiella and Acinetobacter baumannii among the two study groups. This finding makes it difficult to extrapolate the data to our patient populations with significantly lower rates of multidrugresistant infections. Third, the mortality rates of both groups were much higher than their 
calculated SAPS II scores would suggest. This may be accounted for by their incorrect methodology for calculating a SAPS II score. Fourth, as the author's state, the large difference in mortality between groups does not exclude the possibility of a chance finding or contributions from unknown confounding factors. Lastly, this was a single center study. It is well documented in the statistical literature that single center studies show larger effect size $(2,3)$ When single center studies are reproduced into multicenter studies, the effect size is often less and occasionally nonexistent. This does not however negate the importance of this study.

This appropriately designed phase 2 trial of targeted heart rate control for volume resuscitated septic shock patients with persistent tachycardia demonstrated a large mortality benefit without obvious adverse effects. The use of beta blockade during septic shock represents an exciting and novel treatment modality that warrants additional study to confirm these results. In the interim, clinicians should carefully consider the merits of early adoption in appropriately selected patients. While no clear disadvantages were identified in this well-done, single-center trial, the literature would caution us not to overestimate the early findings of promising new treatments.

Christopher Strawter MD, Cristine Berry MD, Christian Bime MD and Joe Gerald PhD University of Arizona

Tucson, AZ

\section{References}

1. Singer M. Catecholamine treatment for shock-equally good or bad? Lancet. 2007;370(9588):636-7. [CrossRef] [PubMed]

2. Dechartres A, Boutron I, Trinquart L, Charles P, Ravaud P. Single-center trials show larger treatment effects than multicenter trials: evidence from a meta-epidemiologic study. Ann Intern Med. 2011;155(1):39-51. [CrossRef] [PubMed]

3. Ioannidis JPA. Why most discovered true associations are inflated. Epidemiology. 2008; 19(5):640-8. [CrossRef] [PubMed] 